International Journal on Artificial Intelligence Tools Vol. 14, No. 5 (2005) 717-731

(C) World Scientific Publishing Company

\title{
A COLLAPSING METHOD FOR THE EFFICIENT RECOVERY OF OPTIMAL EDGES IN PHYLOGENETIC TREES
}

\author{
MICHAEL HU \\ Dana-Farber Cancer Institute \\ 44 Binney St., Sm1058, Boston, MA 02115, USA \\ mike_hu@dfci.harvard.edu \\ PAUL KEARNEY \\ Caprion Pharmaceuticals, 7150 Alexander Fleming \\ Montreal, Quebec H4S 2C8, Canada \\ pkearney@caprion.com \\ JONATHAN BADGER \\ The Institute for Genomic Research \\ 9712 Medical Center Drive, Rockville, MD 20850, USA \\ jbadger@tigr.org
}

\begin{abstract}
As the amount of sequencing efforts and genomic data volume continue to increase at an accelerated rate, phylogenetic analysis provides an evolutionary context for understanding and interpreting this growing set of complex data. We introduce a novel quartet based method for inferring molecular based phylogeny called hypercleaning* $\left(\mathrm{HC}^{*}\right)$. The $\mathrm{HC}^{*}$ method is based on the hypercleaning (HC) technique, ${ }^{2}$ which possesses an interesting property of recovering edges (of a phylogenetic tree) that are best supported by the witness quartet set. $\mathrm{HC}^{*}$ extends $\mathrm{HC}$ in two regards: (i) whereas $\mathrm{HC}$ constrains the input quartet set to be unweighted (binary valued), $\mathrm{HC}^{*}$ allows any positive valued quartet scores, enabling more informative quartets to be defined. (ii) $\mathrm{HC}^{*}$ employs a novel collapsing technique which significantly speeds up the inference stage, making it empirically on par with quartet puzzling in terms of speed, while still guaranteeing optimal edge recovery as in HC. This paper is primarily aimed at presenting the algorithmic construction of $\mathrm{HC}^{*}$. We also report some preliminary studies on an implementation of $\mathrm{HC}^{*}$ as a potentially powerful approximation scheme for maximum likelihood based inference.

Details of proofs can be found in report at: (www.michaelhu.com/reports/ mmath_thesis.pdf).
\end{abstract}

\section{Introduction}

Inferring phylogenetic trees on molecular sequences has wide applications in biology, from analyzing the evolutionary history of AIDS, ${ }^{8}$ to detecting regulatory elements in genetic sequences. ${ }^{1}$ The problem of phylogenetic inference is considered hard from both a biological and computational perspective. Many biologists believe that 
maximum likelihood (ML) based methods are the best vehicle for conducting phylogenetic analysis. ${ }^{9,14}$ ML methods are, however, NP hard optimization problems requiring expensive parameter estimation and topology searching procedures, and is computationally impractical on large data sets. ${ }^{3}$ To date, many approximation methods for ML based inference have been proposed such as quartet puzzling, ${ }^{16}$ PAUP parsimony, ${ }^{12}$ and structural EM. ${ }^{5}$ We present a novel quartet based method called hypercleaning* $\left(\mathrm{HC}^{*}\right) . \mathrm{HC}^{*}$ is based on the quartet method paradigm ${ }^{6,2,7}$ and is guaranteed to return the best supported edges with respect to the quartet witness set. $\mathrm{HC}^{*}$ is computationally efficient and runs with an empirical running time on the order of quartet puzzling. ${ }^{16}$ Unlike other heuristic quartet methods such as quartet puzzling or the Short Quartet method, ${ }^{4} \mathrm{HC}^{*}$ is guaranteed to return those edges best supported by the witness quartet set. This effectively focuses the problem of phylogenetic inference under the quartet paradigm onto accurate construction of the witness quartet set (i.e. phylogenetic analysis on input size four). By using sophisticated ML based methods for inferring the witness quartet set, $\mathrm{HC}^{*}$ can serve as an effective method for approximating ML.

\section{Methods and Terminologies}

Given an input set $S$ of $n$ sequences (e.g. homologous gene sequences on $n$ organisms), the task is to infer the true phylogeny or tree $T$ that captures the evolutionary relationship of these $n$ extant leaf sequences. Note that the leaves of $T$ are labelled by $S$, and the internal nodes of $T$ represents speciation events on unobservable, ancestral objects. One class of phylogenetic inference methods are the quartet based methods. Most quartet based methods have two stages: (1) the witness quartet set inference stage and (2) the recombination stage. Given an input set $S$ of size $n$, the first stage consists of inferring all $\left(\begin{array}{l}n \\ 4\end{array}\right)$ unique quartet topologies called a witness quartet set, denoted by $W$. The second recombination stage, takes the inferred quartet topologies in $W$ (i.e. hypothesis to sub-problems on input size four) and combine them into a hypothesis tree $T^{\prime}$, estimating the underlying true tree $T$. Figure 1 shows the high level overview of quartet based methods.

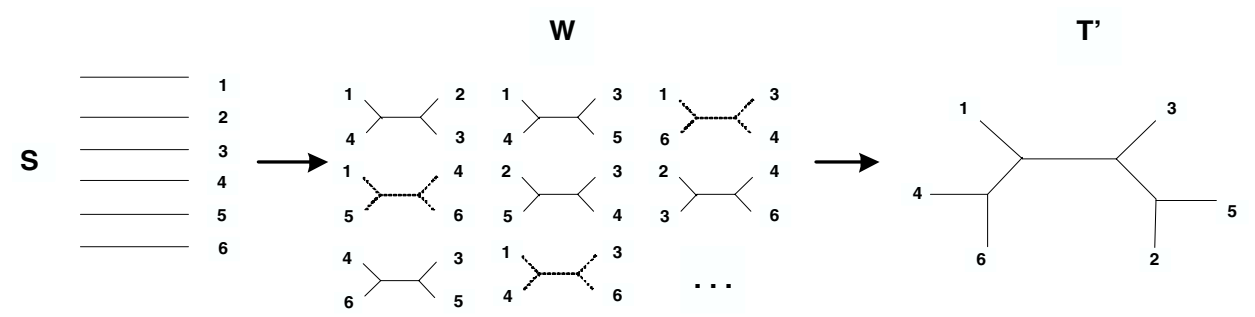

Fig. 1. Overview of quartet based methods. First stage takes the input set $S$, and employs some existing inference method(s) to construct the witness quartet set $W$, shown partially. Then the quartet method pieces the information from $W$ into a hypothesis tree $T^{\prime}$. Note that not all quartets in set $W$ are guaranteed to be compatible with the estimate tree $T^{\prime}$, as the ones in dashes. 

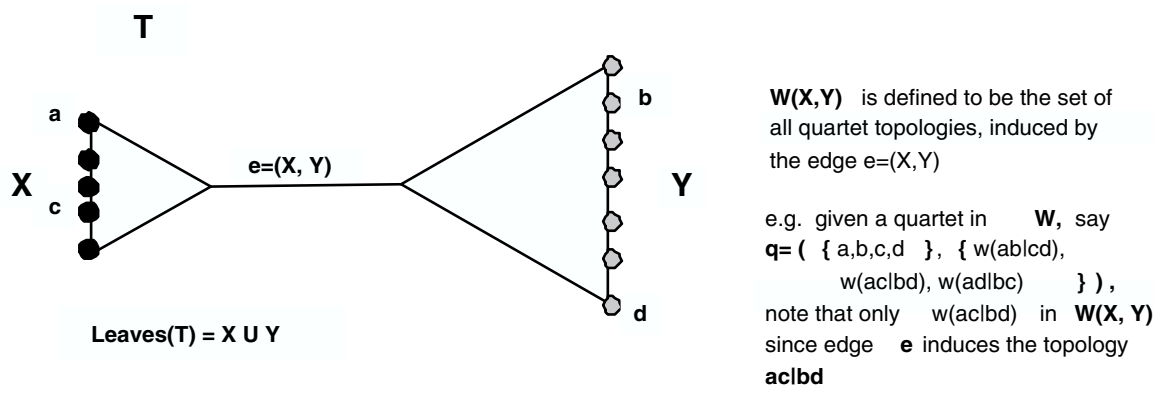

Fig. 2. An edge $e$ of an unrooted binary tree $T$, and the quartet topologies in the witness quartet set $W$ induced by $e$.

\section{Definition 1. A Quartet}

A quartet $q$ in witness quartet set $W$, consists of the pair $(\{a, b, c, d\},\{w(a b \mid c d)$, $w(a c \mid b d), w(a d \mid b c)\})$ where $a, b, c, d \in S$ and $w(a d \mid b c) \in \Re$ is the score (or support) that topology $a b \mid c d$ is contained in the underlying phylogeny $T$. Note that on some figures and examples to follow, we limit ourselves to binary quartets, where on a given quartet all the support falls onto one topology (with score of 1), and the other topologies have scores of 0 . A quartet topology $a b \mid c d$ indicates that the path in $T$ connecting leaves $a, b$ is disjoint from the path connecting $c, d$.

The $\mathrm{HC}^{*}$ algorithm has two major components: the edge inference component $\left(\mathrm{HC}_{E}^{*}\right)$ and the collapsing component $\left(\mathrm{HC}_{C O L L A P S E}^{*}\right)$. For the remainder of this section, we introduce the supporting concepts and definitions, deferring the actual algorithm until the next section.

$\mathrm{HC}^{*}$ on input set $S$ and its inferred witness quartet set $W$, is guaranteed to return the set of edges best supported by $W$. We define an edge $e$, and the distance (i.e. lack of support) of an edge $e$ by $W$ as follows:

Definition 2. Edge (bipartition) of a phylogeny

An edge $e$ in an evolutionary tree $T$ is defined by the bipartition $(X, Y)$ where $X, Y$ denotes the leaves set of the two disjoint sub-trees of $T$ resulting from removing $e$. Note that $X \cup Y=S$, and $X \cap Y=\{\}$. The left part of Figure 2 illustrates.

Definition 3. Distance function of an edge e

Given an edge $e=(X, Y)$, its distance or quartet error with respect to $W$, is given by

$$
\sigma(e, W)=\frac{\sum_{a b \mid c d \in W(X, Y)} w(a c \mid b d)+w(a d \mid b c)}{\left(\begin{array}{c}
|X| \\
2
\end{array}\right)\left(\begin{array}{c}
|Y| \\
2
\end{array}\right)}
$$

where $W(X, Y)$ are the topologies of quartet entries in $W$ induced by the edge $e=(X, Y)$. The denominator is the normalizing factor, since note that there are $\left(\begin{array}{c}|X| \\ 2\end{array}\right)\left(\begin{array}{c}|Y| \\ 2\end{array}\right)$ quartet topologies induced by edge $e$. Figure 2 illustrates. 
The edge recovery component ${ }^{\mathrm{a}}$ of $\mathrm{HC}^{*}$, denoted $\mathrm{HC}_{E}^{*}$, has the following interface:

$$
\operatorname{Best}(m, W) \longleftarrow \mathrm{HC}_{E}^{*}(m, W)
$$

whereby $\mathrm{HC}_{E}^{*}$ takes in a witness quartet set $W$ on the input taxa set $S$, and input parameter $m \in \aleph$ and returns the set of best supported edges $\operatorname{Best}(m, W)$, defined as follows:

Definition 4. Best supported edge set $\operatorname{Best}(m, W)$

Given input parameter $m$, and the witness quartet set $W$, the set of best supported edges is given by:

$$
\operatorname{Best}(m, W)=\left\{(X, Y):: \sigma((X, Y), W)<\frac{2 m}{|X||Y|}\right\}
$$

Similar to the $\mathrm{HC}$ algorithm, the $\mathrm{HC}^{*}$ component for constructing the set $\operatorname{Best}(m, W)$ has a parameterized polynomial upper bound of $O\left(n^{3} f(2 m)\right.$ ) (see Ref. 2 for proof), where

$$
f(m)=4 m^{2}(1+2 m)^{4 m} .
$$

On lower bounded values of $m$, this yields a polynomial time algorithm. But on practical datasets, the value of $m$ required such that set $\operatorname{Best}(m, W)$ contains enough compatible edges for constructing a fully resolved tree ${ }^{\mathrm{b}}$ renders $\mathrm{HC}$ computationally intractable. Here we say a set of edges are compatible if they can all exist in the same binary tree.

The $\mathrm{HC}^{*}$ method addresses the aforementioned efficiency problem through the use of a time/memory tradeoff collapsing mechanism, denoted $\mathrm{HC}_{\text {COLLAPSE }}^{*}$. In essence, the collapsing mechanism enables $\mathrm{HC}^{*}$ to run at a low value of $m$, returning the set $\operatorname{Best}(m, W)$ of edges. A subset of compatible edges from such a set will induce an unresolved tree, or a cluster tree. The collapsing mechanism will then collapse all but one of the clusters in this unresolved tree. The resulting single cluster can be viewed as simply a star topology on a set of leaves and collapsed nodes. Moreover this cluster set has fewer vertices than the original input set $S$. We can then run $\mathrm{HC}^{*}$ on this smaller cluster set, and attempt to resolve edges that lie in that cluster by using a higher value of $m$. In other words, we reduce the input size $n$, such that we can raise the input parameter $m$, allowing more edges to be resolved without accruing the high cost of added computational time. Figure 3 shows the idea.

The collapsing mechanism guarantees no information loss during the collapse, such that any edges recovered by $\mathrm{HC}^{*}$ under a collapsed cluster, will have the

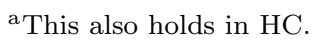

${ }^{\mathrm{b}}$ Given the input set $S$ of size $n$, the necessary condition for $\mathrm{HC}^{*}$ to return a fully resolved (unrooted) tree, is that set $\operatorname{Best}(m, W)$ contains at least $n-3$ compatible edges. 

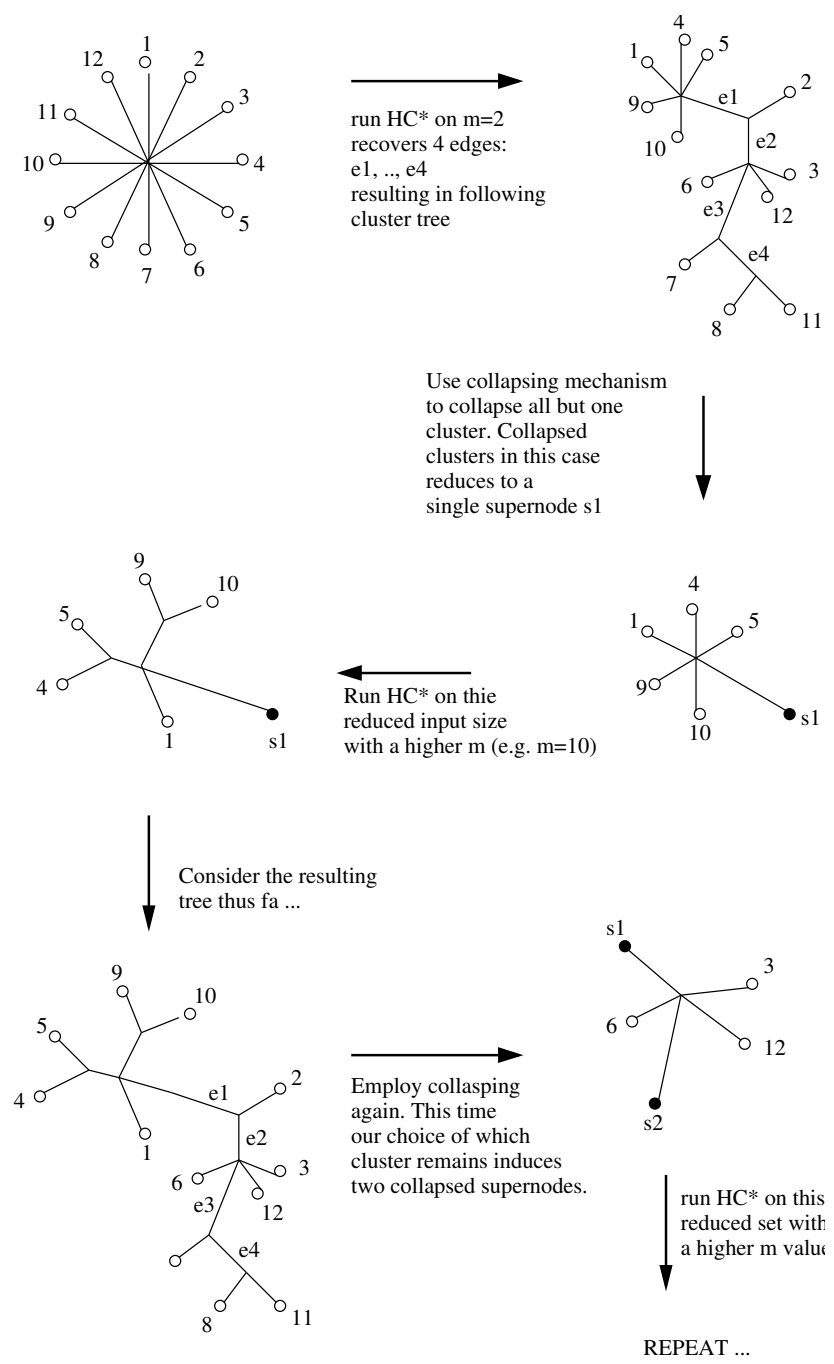

Fig. 3. Running $\mathrm{HC}^{*}$ with low values of $m$ and further resolution of the unresolved tree by recursively collapsing the problem into smaller instances and then running $\mathrm{HC}^{*}$ with higher values of $m$ on these smaller problems. Here a solid dot represents a collapsed sub-tree and an open circle represents an original input leaf.

same score with respect to $W$ as under no collapsing with the original input set $S$, by raising the value of $m$ to the necessary level. This will be formally defined in Property 1 later on.

Definition 5. Cluster Tree

A cluster tree $T$ is a tree consisting of edges and vertices, where each vertex is either a leaf, an internal vertex (i.e. non-leaf), or a supernode. Note that a regular tree is a cluster tree whose vertices are either leaves or internal vertices. 
Definition 6. Cluster

Given a cluster tree $T$, a cluster $C_{i} \in T$ is the set of vertices consisting of exactly one internal vertex, along with its degree- 1 neighboring vertices (i.e. leaves and supernodes). The leaves of a cluster $C_{i}$ are denoted Leaves $\left(C_{i}\right)$, and the supernodes (see following definition) in $C_{i}$ are denoted SuperNodes $\left(C_{i}\right)$. Thus we have $C_{i}=$ an internal node $\cup \operatorname{SuperNodes}\left(C_{i}\right) \cup \operatorname{Leaves}\left(C_{i}\right)$. An internal vertex with no adjacent leaves or supernodes is a trivial cluster. Let $C l u(T)$ be the set of all clusters in $T$.

Definition 7. Supernode

A supernode $\mathbf{c}$ of a cluster tree $T$, is a degree-1 non-leaf vertex, denoting some collapsed cluster $C_{i}$.

Definition 8. Cluster Collapse

Given a cluster tree $T$ and cluster $C_{i} \in T$, the cluster can be collapsed into a single node $\mathbf{c}_{\mathbf{i}}$, only if the resulting node $\mathbf{c}_{\mathbf{i}}$ is a degree- 1 vertex in the remaining tree. After the collapse, the tree remains a connected tree, but has one more supernode and one less cluster.

Figure 5 illustrates a valid and an invalid cluster collapse.

Definition 9. Full Collapse of a tree : Full $\bullet\left(T \mid C_{i}\right)$

Given a (cluster) tree $T$, its full collapse with respect to cluster $C_{i}$, denoted Full $\bullet\left(T \mid C_{i}\right)$ is the ordering of all the clusters $C_{j} \neq C_{i} \in C l u(T)$, and the subsequent collapse in turn of these ordered clusters. The ordering must be valid, in the sense that each cluster, on its turn to collapse, must be collapsible as in previous definition, with respect to $T$.

The result of the sequence of collapses is a single cluster $C_{i}^{*}=C_{i} \cup\left\{\mathbf{s}_{\mathbf{1}}, \ldots, \mathbf{s}_{\mathbf{k}}\right\}$, $k<|S|$, and the corresponding tree topology on $C_{i}^{*}$ is simply the star topology.

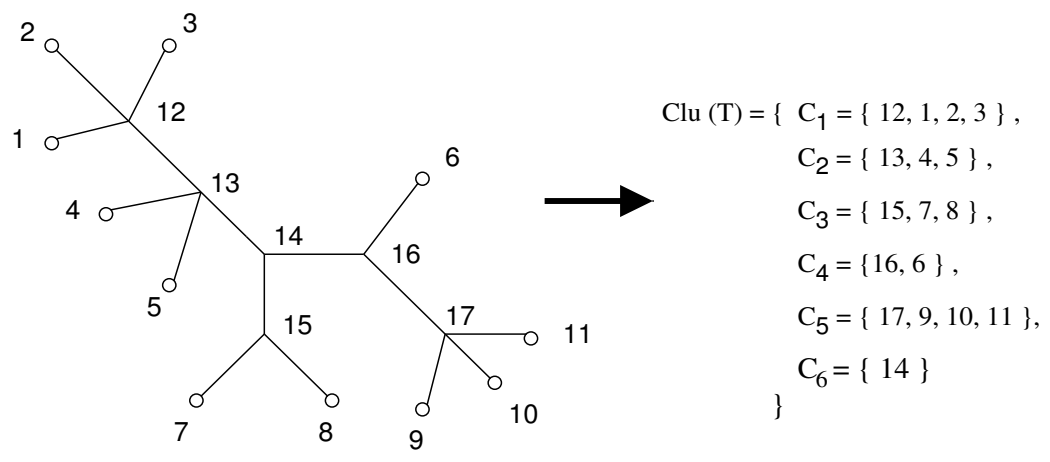

Fig. 4. An unresolved cluster tree, with clusters $C_{1}, \ldots, C_{6} . C_{6}$ is a trivial cluster. 
(1)

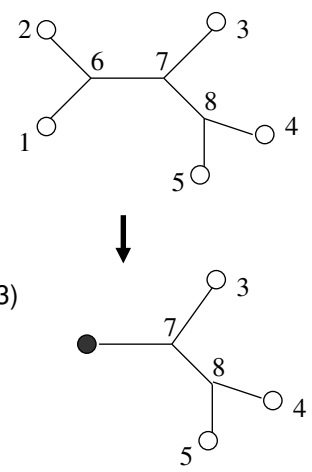

(2)

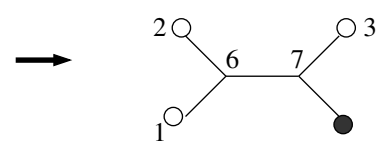

(4)

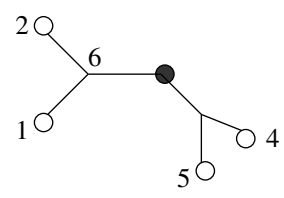

Fig. 5. The cluster tree (1) has 3 clusters: $C_{6}=\{6,1,2\}, C_{7}=\{7,3\}$, and $C_{8}=\{8,4,5\}$. Tree (2)results from the valid collapse of cluster $C_{8}$ in tree (1), since the resulting vertex satisfies the definition of a supernode. (3) is the valid collapse of cluster $C_{6}$ from (1). In tree (4), the collapse of cluster $C_{7}$ is not valid since the resulting vertex does not satisfy the definition of a supernode.

Definition 10. Size and Cardinality of Vertices

Given a vertex $s$, we define its size recursively as

$$
\operatorname{Size}(s)= \begin{cases}1, & s \text { is a leaf or intern. vertex } \\
\sum_{v \in C_{s}} \operatorname{Size}(v) & \begin{array}{l}
s \text { is a supernode, } C_{s} \\
\text { is the cluster collapsed to } s
\end{array}\end{cases}
$$

The cardinality of a vertex $s$, denoted $|s|$ is 1 if $s$ is a leaf or an internal vertex, and the number of elements in its corresponding cluster if $s$ is a supernode.

The following lemma states that is always possible to correctly collapse the clusters of a cluster tree until only one (pre-determined)cluster remains.

Lemma 1. Generating a valid collapse ordering for Full $\bullet\left(T \mid C_{i}\right)$

Given tree $T$ with $k$ clusters, a valid ordering of the clusters can be obtained by sorting all clusters $\neq C_{i}$ by decreasing distance from $C_{i}$, where the $\operatorname{dist}(C, D)$ between two clusters is simply the number of edges between their closest vertices. Ties in the ordering can be settled arbitrarily.

Proof. Omitted

Definition 11. Expanding a supernode: $\exp (\mathbf{s})$

Consider a supernode $\mathbf{s}$ resulted from collapsing a cluster $C_{s}$. The expansion of $\mathbf{s}$, denoted $\exp (\mathbf{s})$ is a recursive procedure, which returns a set of leaves as follows:

$$
\exp (\mathbf{s})=\left\{\operatorname{Leaves}\left(C_{s}\right) \bigcup_{\forall s^{\prime} \in \operatorname{SuperNode}\left(C_{s}\right)} \exp \left(s^{\prime}\right)\right\}
$$

In the context of collapsing, an edge $e=(X, Y)$ defined by its bipartition of vertices also becomes more general. In a normal tree $T$, an edge $e=(X, Y)$ consists 
of two disjoint but pairwise complete sets on the leaves $S$. In a cluster tree $T_{C}$, an edge $e^{\prime}=\left(X^{\prime}, Y^{\prime}\right)$ also consists of two disjoint, pairwise complete sets on all degree-1 vertices in $T_{C}$ (i.e. leaves and supernodes).

Definition 12. Expansion of an edge e in a cluster tree

Given an edge $e=(X, Y)$ in cluster tree $T_{C}$, its full expansion, denoted $\operatorname{Exp}(e=$ $(X, Y))$ returns the edge $e^{\prime}=\left(X^{\prime}, Y^{\prime}\right)$ where $X^{\prime}, Y^{\prime}$ is a bipartition on the original leaves set $S$. Formally, the $\operatorname{Exp}(e=(X, Y))$ is recursively defined as follows:

$$
\begin{aligned}
\operatorname{Exp} & (e=(X, Y))=\left(X^{\prime}, Y^{\prime}\right) \\
& =\left(\text { Leaves }(X) \bigcup_{s \in \text { SuperNode }(X)} \exp (s), \text { Leaves }(Y) \bigcup_{s \in \text { SuperNode }(Y)} \exp (s)\right)
\end{aligned}
$$

Having the above definitions on a cluster tree, collapsing, and expanding under a cluster tree, we are ready to formally state the definition of what it means for $\mathrm{HC}^{*}$ to achieve information loss-less collapsing of the cluster tree into a single cluster, and subsequent resolution of edges on that cluster.

Property 1. Information loss-less edge recovering under collapsing Given input leaves set $S$ and its witness quartet set $W$, assume that $\mathrm{HC}^{*}$ is at some stage of collapsing some semi-resolved cluster tree into a star topology tree, whose leaves are on the cluster set:

$$
C=\left\{l_{1}, l_{2}, \ldots, l_{k}, \mathbf{s} \mathbf{1}, \ldots, \mathbf{s p}\right\}
$$

consisting of leaves and supernodes. We wish to construct the quartet set $W^{*}$ on the vertices of $C$, such that running $\mathrm{HC}^{*}$ on input $C$ using $W^{*}$, will return the edge set $\operatorname{Best}\left(m, W^{*}\right)$ with the following accuracy guarantee:

$$
\forall \text { edges } e \in \operatorname{Best}\left(m, W^{*}\right):: \sigma\left(e, W^{*}\right)=\sigma(\operatorname{Exp}(e), W) .
$$

Such a quartet set $W^{*}$ then satisfies the information lossless property of the cluster $C$.

\section{Algorithms}

The $\mathrm{HC}^{*}$ algorithm has two main components, (i) the edge recovery component, denoted $\mathrm{HC}_{E}^{*}$ and a collapsing component $\mathrm{HC}_{C O L L A P S E}^{*}$. Figure 6 shows the high level flow of $\mathrm{HC}^{*}$.

\subsection{The $H C_{E}^{*}$ component}

This section describes the algorithmic construction of the $\mathrm{HC}_{E}^{*}$ component. $\mathrm{HC}_{E}^{*}$ on input vertex set $C$ of size $n$, and integer parameter $m$ produces the edge set $\operatorname{Best}(m, W)$ which we redefine as follows:

$$
\operatorname{Best}(m, W)=\left\{(X, Y) \mid \sigma(W,(X, Y))<\frac{2 m}{\operatorname{Size}(X) \operatorname{Size}(Y)}\right\} .
$$




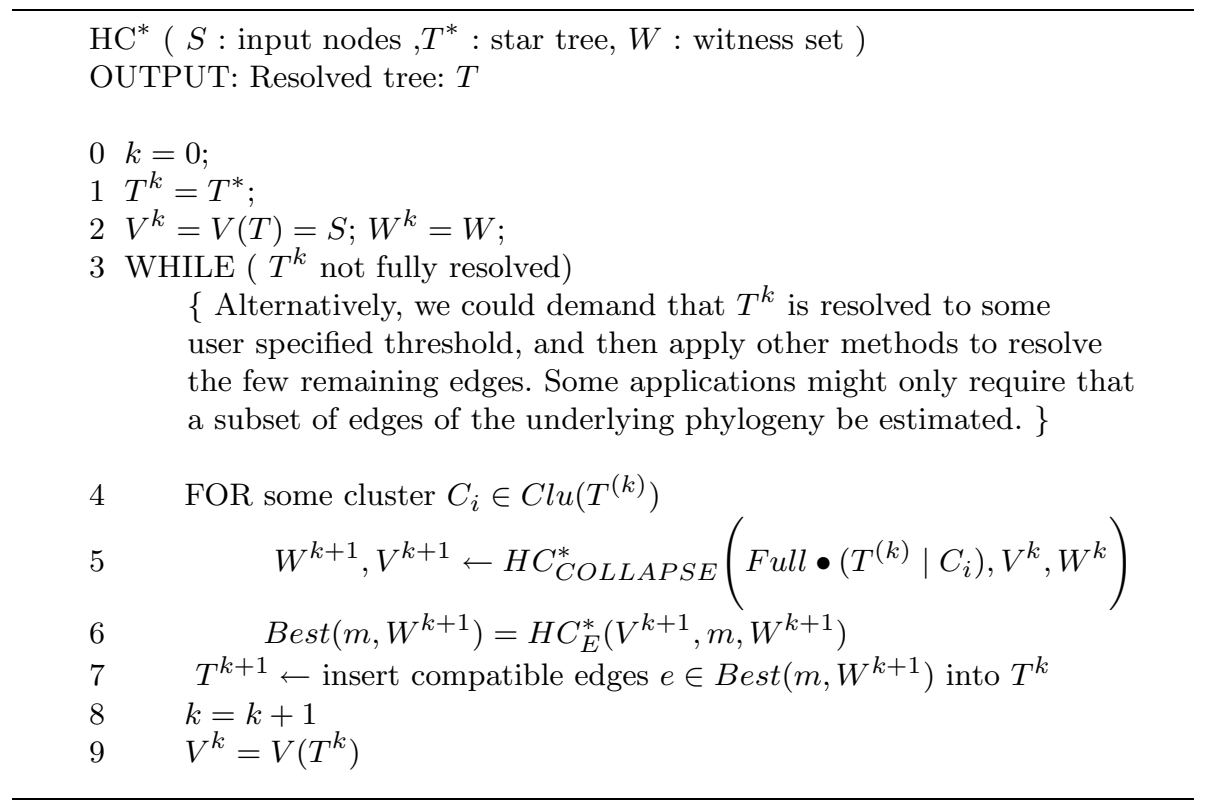

Fig. 6. High level view of the $\mathrm{HC}^{*}$ algorithm.

Notice that the above definition is a slight modification of our earlier definition (2), since in this more general setting, our input node set $C$ might have a mix of leaves and supernodes.

The $\mathrm{HC}_{E}^{*}$ produces the set $\operatorname{Best}(m, W)$ by first constructing for all pairs $x, y \in$ $C$, the set:

$$
\operatorname{Best}_{x y}\left(m, W_{k}\right)=\left\{(X, Y):: \sum_{a x \mid b y \in W(X, Y)} \frac{w(a y \mid b x)+w(a b \mid x y)}{\operatorname{Size}(x) \operatorname{Size}(y)}<m\right\}
$$

where $W_{k}$ is the subset of $W$ induced by the sequence of vertices: $S_{k}=$ $\left\{x, y, v_{1}, v_{2}, \ldots, v_{k-2}\right\} \subseteq C, k<n$ and $\operatorname{Size}(x)$ as defined in the previous section. The construction of this set is iterative on $k=1, \ldots, n$. Moreover any trivial edges with either zero or one element in either of its two partitions belongs to $\operatorname{Best}_{x y}\left(m, W_{k}\right)$, although trivial edges induces no quartets. Note that $\operatorname{Best}_{x y}(m, W)=\operatorname{Best}_{x y}\left(m, W_{k}\right)$ when $k=n$.

Procedure 1. Constructing $\operatorname{Best}_{x y}\left(m, W_{k}\right)$ for $k=1, \ldots, n$

If $k=1$ then Best $_{x y}\left\{m, W_{k}\right\}=\emptyset$, since $W_{1}$ does not contain any bipartitions.

Else If $k=2$, then Best ${ }_{x y}\left\{m, W_{k}\right\}=\{(\{x\},\{y\})\}$.

Else If $k \geq 3$, then

$$
\operatorname{Best}_{x y}\left(m, W_{k}\right)=\forall \text { edges } e \in L_{x y} \cup R_{x y} \text { satisfying Eq. (7) }
$$


where

$$
\begin{aligned}
& L_{x y}=\left\{\left(X \cup\left\{s_{k}\right\}, Y\right) \mid(X, Y) \in \text { Best }_{x y}\left(W_{k-1}, m\right)\right\} \\
& R_{x y}=\left\{\left(X, Y \cup\left\{s_{k}\right\}\right) \mid(X, Y) \in \text { Best }_{x y}\left(W_{k-1}, m\right)\right\}
\end{aligned}
$$

and the vertex $\left\{s_{k}\right\}$ is the kth element drawn from the sequence $S_{k}=$ $\left\{x, y, v_{1}, v_{2}, \ldots, v_{k-2}\right\} \subseteq C$.

Theorem 1. Given input $S$ and $W$, the set constructed under the above procedure, $B_{e s t}\left(m, W_{k}\right)$, for $k=n$, satisfies the definition of $\operatorname{Best}_{x y}(m, W)$ as given in Equation (7).

Proof. Omitted

Procedure 2. Constructing the set $\operatorname{Best}\left(m, W_{k}\right)$

On input set $S$ of $n$ leaves, the set $\operatorname{Best}\left(m, W_{k}\right)$, is defined iteratively from $\operatorname{Best}\left(m, W_{k-1}\right)$, for $2 \leq k \leq n$, where $\operatorname{Best}(m, W)=\operatorname{Best}\left(m, W_{n}\right) . W_{k}$ is the subset of quartets of $W$ induced by the subset of leaves $S_{k}=\left\{v_{1}, v_{2}, \ldots, v_{k}\right\} \subseteq S$. If $k=1$ then $\operatorname{Best}\left(m, W_{1}\right)=\emptyset$.

If $k \geq 2$ then

$$
\operatorname{Best}\left(m, W_{k}\right)=\forall \text { edges } e \in L \cup R \cup M \text { satisfying Eq. (6) }
$$

where the sets $L, R, M$ are constructed as follows:

$$
\begin{aligned}
L & =\left\{\left(X \cup\left\{s_{k}\right\}, Y\right)::(X, Y) \in \operatorname{Best}\left(m, W_{k-1}\right)\right\} \\
R & =\left\{\left(X, Y \cup\left\{s_{k}\right\}\right)::(X, Y) \in \operatorname{Best}\left(m, W_{k-1}\right)\right\} \\
M & =\bigcup_{x \in S_{k-1}} \operatorname{Best}_{x s_{k}}\left(m, W_{k}\right)
\end{aligned}
$$

Theorem 2. Given input $S$ and $W$, the resulting set from Procedure 2, $\operatorname{Best}\left(m, W_{k}\right)$ for $k=n$, defines the set $\operatorname{Best}(m, W)$ as given by Equation (6).

Proof. Omitted

Procedures 1 and 2 in essence describe the iterative construction of the set $\operatorname{Best}(m, W)$. The efficiency is that at each stage for $1 \leq k \leq n$, the cardinality of the set $\operatorname{Best}\left(m, W_{k}\right)$ is inherently constrained by the size of the sets $\operatorname{Best}\left(m, W_{k-1}\right)$ and $\operatorname{Best}_{x y}\left(m, W_{k}\right)$, with the guarantee that $\operatorname{Best}(m, W)$ does not miss any edges. This in essence constrains the edge space to be searched through, whereas the naive search on $n$ leaves involves searching through $2^{n}$ edges.

\subsection{The HCOLLAPSE component}

Suppose we run $\mathrm{HC}^{*}$ where we have already done $k$ number of collapses (i.e. the WHILE loop in Figure 6, resulting in the current cluster tree $T_{C}^{(k)}$, on vertex set $V^{(k)}$ and the quartet set $W^{(k)}$ on $V^{k}$. Assume that the quartet set $W^{k}$ satisfies the 
loss-less condition. Consequently for any edge $e=(X, Y)$ on $V^{k}$, we have:

$$
\sigma\left(e=(X, Y), W^{k}\right)=\sigma(\operatorname{Exp}(e), W)
$$

where $W$ is the quartet set on the original input set $S$. Now $\mathrm{HC}^{*}$ will choose a cluster $C_{i} \in T_{C}^{k}$ and perform Full $\bullet\left(T_{C}^{k} \mid C_{i}\right)$, effectively creating a single cluster. The following procedure describes how one produces the witness quartet set $W^{k+1}$ on the resulting single cluster $V^{k+1}$ such that the loss-less condition is preserved.

Procedure 3. $W^{k+1}, V^{k+1} \leftarrow \mathrm{HC}_{C O L L A P S E}^{*}\left(F u l l \bullet\left(T_{C}^{k} \mid C_{i}\right), V^{k}, W^{k}\right)$

Given some unresolved cluster tree $T_{C}^{k}$ on vertex set $V^{k}$ with witness set $W^{k}$, we wish to collapse all the clusters of $T_{C}^{k}$ except $C_{i}$. The collapse ordering is defined by Full $\bullet\left(T_{C}^{k} \mid C_{i}\right)$. Without loss of generality, suppose the collapse ordering is given by:

$$
C_{1}, C_{2}, \ldots, C_{i-1}, C_{i+1}, \ldots, C_{q}
$$

The procedure is governed by the aforementioned collapse ordering and can be characterized by a sequence of corresponding function calls to $\mathrm{HC}_{C O L L A P S E}^{*}$ :

$$
\begin{gathered}
W_{(1)}^{k}, V_{(1)}^{k} \leftarrow \mathrm{HC}_{\text {COLLAPSE}}^{*}\left(V^{k}, W^{k}, C_{1}\right) \\
W_{(2)}^{k}, V_{(2)}^{k} \leftarrow \operatorname{HC}_{\text {COLLAPSE }}^{*}\left(V_{(1)}^{k}, W_{(1)}^{k}, C_{2}\right) \\
\ldots \\
W^{k+1}, V^{k+1} \leftarrow \operatorname{HC}_{\text {COLLAPSE }}^{*}\left(V_{(q-1)}^{k}, W_{(q-1)}^{k}, C_{q}\right)
\end{gathered}
$$

The next procedure defines the actual algorithm for:

$$
\mathrm{HC}_{C O L L A P S E_{j+1}}^{*}\left(V_{(j)}^{k}, W_{(j)}^{k}, C_{j+1}\right)
$$

for $j=0, \ldots, q-1$. Note that $V^{0}, W^{0}$ corresponds to the original input vertex set and its quartet set $V, W$.

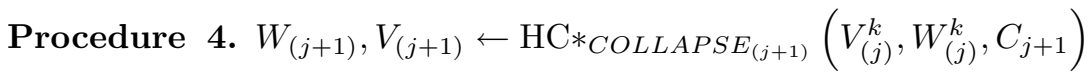

Assume we are currently in the $k$-th iteration of $F u l l \bullet\left(T_{C} \mid C_{i}\right)$, and have performed $q$ cluster collapses thus far, resulting in vertex sets $V_{(j)}$, and quartet sets $W_{(j)}$, for $j=0, \ldots, q-1$. Moreover we assume that $W_{(j)}$ on $V_{(j)}$ satisfies the information loss-less precondition. Assume we want to collapse a cluster $C \neq C_{i} \subseteq V_{(j)}$ into supernode $\mathbf{s}$, such that we have $V_{(j+1)}=V_{(j)}-C+\mathbf{s}$. Thus, we construct an updated quartet set $W_{(j+1)}$ on $V_{(j+1)}$ as follows, to satisfy the loss-less precondition:

Consider the following combinations of quartet: $(a, b, c, d) \in V_{(j+1)}=V_{(j)}-C+\mathbf{s}$, where these vertices do not have to be all distinct ${ }^{\mathrm{c}}$

${ }^{\mathrm{C}}$ Quartets can be of the form $(a, a, b, c)$ since $a$ might be a supernode which semantically represents collapsed leaves and or other supernodes. As such when we consider the distance score for a quartet topology $a, a \mid b, c$, we take into account the following alternative: $a, b \mid a, c$ which represents all quartets of the form $a_{1}, b \mid a_{2}, c$, where $a_{1}, a_{2}$ are two collapsed vertices in supernode $a$. A quartet of the form $(a, a, a, b)$ is impossible since no supernode can span across an edge. 
C1 $(a, b, c, d)$, where $a \neq b \neq c \neq d \in V_{(j+1)}-\mathbf{s}$ :

For all such quartets, assign:

$$
\begin{aligned}
& w_{(j+1)}(a b \mid c d)=w_{(j)}(a b \mid c d) \\
& w_{(j+1)}(a c \mid b d)=w_{(j)}(a c \mid b d) \\
& w_{(j+1)}(a d \mid b c)=w_{(j)}(a d \mid b c)
\end{aligned}
$$

C2 $(a, a, c, d)$, where $a \neq c \neq d \in V_{(j+1)}-\mathbf{s}$ :

For quartets of this form, assign:

$$
w_{(j+1)}(a c \mid a d)=w_{(j)}(a c \mid a d)
$$

C3 $(a, b, c, c)$, where $a \neq b \neq c \in V_{(j+1)}-\mathbf{s}$ :

For quartets of this form, assign:

$$
w_{(j+1)}(a c \mid b c)=w_{(j)}(a c \mid b c)
$$

C4 $(a, a, b, b)$, where $a \neq b \in V_{(j+1)}-\mathbf{s}$ :

For quartets of this form, assign:

$$
w_{(j+1)}(a b \mid a b)=w_{(j)}(a b \mid a b)
$$

C5 $(a, a, \mathbf{s}, \mathbf{s})$, where $a \in V_{(j+1)}-\mathbf{s}$

For these quartets, assign:

$$
w_{(j+1)}(a b \mid a b)=\sum_{c, d \in C} w_{(j)}(a c \mid a d)+\sum_{c \in C} w_{(j)}(a c \mid a c)
$$

C6 $(a, b, c, \mathbf{s})$, where $a \neq b \neq c, a, b, c \in V_{(j+1)}-\mathbf{s}$ :

For quartets of this form, assign:

$$
\begin{aligned}
& w_{(j+1)}(a b \mid c \mathbf{s})=\sum_{d \in C} w_{(j)}(a b \mid c d) \\
& w_{(j+1)}(a c \mid b \mathbf{s})=\sum_{d \in C} w_{(j)}(a c \mid b d) \\
& w_{(j+1)}(b c \mid a \mathbf{s})=\sum_{d \in C} w_{(j)}(b c \mid a d)
\end{aligned}
$$

C7 $(a, b, \mathbf{s}, \mathbf{s})$, where $a \neq b \in V_{(j+1)}-\mathbf{s}$

For these quartets, assign:

$$
w_{(j+1)}(a \mathbf{s} \mid b \mathbf{s})=\sum_{c \in C} w_{(j)}(a c \mid b c)+\frac{1}{2} \sum_{c, d \in C}\left(w_{(j)}(a c \mid b d)+w_{(j)}(a d \mid b c)\right)
$$

C8 $(a, a, b, \mathbf{s})$, where $a \neq b \in V_{(j+1)}-\mathbf{s}$

For these quartets, assign:

$$
w_{(j+1)}(a b \mid a \mathbf{s})=\sum_{d \in C} w_{(j)}(a b \mid a d)
$$


Theorem 3. Given procedures 3 and 4 construction of $\operatorname{HC}_{C O L L A P S E_{(j+1)}}^{*}\left(V_{(j)}^{k}\right.$, $\left.W_{(j)}^{k}, C_{j+1}\right)$ on the $(j+1)$-th cluster collapse in the sequence of cluster collapses as given by Full $\bullet\left(T_{C} \mid C_{i}\right)$, the resulting quartet set $W_{(j+1)}$ on the updated vertex set $V_{(j+1)}$ satisfies the information loss-less precondition. Note this is performed in the $k$ th iteration of the outer WHILE loop of the $\mathrm{HC}^{*}$ algorithm (see Figure 6).

\section{Proof. Omitted}

\section{Results}

We designed an experiment as a preliminary gauge of the utility of $\mathrm{HC}^{*}$ for approximating the Maximum Likelihood (ML) method under realistic circumstances (i.e. when our assumptions about the model of evolution is only partially correct). In particular we compared $\mathrm{HC}^{*}$ with treepuzzle ${ }^{16}$ (the original implementation of the quartet puzzling algorithm, considered by many to be one of the better ML approximation methods to date). We generated the input sequence sets using simulation on known (ML analyzed) tree topologies, The simulated datasets were generated by first choosing several tree topologies with varying branch length composition, on input leaves sizes: $30,50,75$. We than generated sequence datasets by 'evolving' sequences along these topologies using the HYK model of evolution with perturbations to its various parameters. The sequences were generated using Seq-Gen. ${ }^{11}$

For the tree topologies we chose:

- two 30-taxon tree topologies randomly sampled from the tree of the set of 218 representative prokaryotic sequences from the RDP database: denoted $S S U \_30$, $S S U \_30 b$.

- two 50-taxon tree topologies randomly sampled from the RDP prokaryotic representative tree from the RDP database, denoted $S S U \_50, S S U_{\_} 50 b$, as well as the 51-taxon Eutherian tree, denoted Euth51 from Ref. 13.

- one 75-taxon tree, denoted $S S U_{-75}$, sampled from the same source tree.

We then simulated sequences along the topology sets using the HYK model of evolution by perturbing 4 parameters as follows: (i) sequence lengths $(2000,4000)$, (ii) gamma heterogeneity $(0.2,0.5)$, (iii) branch lengths scaling factor $(1,4,10)$, and (iv) transversion/transition ratio $(2,4)$, effectively generating 24 sequence sets per tree topology. ${ }^{\mathrm{d}}$ Using these simulated sequences as input, we ran both treepuzzle ${ }^{16}$ and $\mathrm{HC}^{*}$ where the witness quartet set ${ }^{\mathrm{e}}$ was inferred using fastDNAml. ${ }^{10}$ With both fastDNAml and treepuzzle, we ran the software on default settings, such that the assumptions on the model of evolution is 'incorrect' with respect to the generated sequences. We then proceed to test the accuracy and robustness of the inference

${ }^{\mathrm{d}}$ On the 75 taxon tree we simulated only 16 sequences, by omitting values 4 on branch scaling parameter due to heavy computations.

e The quartets were unweighted, where a score of 1 was assigned to the highest scoring topology and 0 to the two alternative topologies on a given quartet. 


\begin{tabular}{|c|c|c|c|c|c|c|}
\hline & SSU_30 & SSU_30b & SSU_50 & SSU_50b & Euth51 & SSU_75 \\
\hline $\begin{array}{c}\text { avg accuracy } \% \\
p \text {-value }\end{array}$ & $65.6 / 74.5$ & $66.1 / 70.8$ & $56.6 / 64.3$ & $46.3 / 46.4$ & $68.1 / 77.5$ & $62.8 / 68.5$ \\
& $4.722 \mathrm{e}-004$ & 0.028 & $4.151 \mathrm{e}-004$ & 0.454 & $4.767 \mathrm{e}-005$ & $9.766 \mathrm{e}-004$ \\
\hline
\end{tabular}

Fig. 7. Average accuracy of treepuzzle / $\mathrm{HC}^{*}$ and the $p$ value on all sampled trees.

methods under such a circumstance when our model of evolution does not match the actual evolutionary process. Figure 6 shows the average accuracy (given as the percentage of edges shared with the true underlying tree), and the $p$-value for testing the statistical significance of accepting the null hypothesis (that the accuracy datapoints on treepuzzle and $\mathrm{HC}^{*}$ came from the same distribution). This was performed using the Wilcoxon paired-sample test (i.e. non-parametric paired t-test) in MATLAB. From Figure 7 we see that $\mathrm{HC}^{*}$ outperforms treepuzzle, in a statistically significant way, on all sequences except those on topology $S S U \_50 b$. This preliminary study shows that $\mathrm{HC}^{*}$ tends to be more robust than treepuzzle on inferring phylogenies when the assumptions on the model of evolution are broken, although more detailed studies need to be conducted to determine the effect of the various parameters on the model of evolution and their effects on the performance of $\mathrm{HC}^{*}$.

On average $\mathrm{HC}^{*}$ runs on the order of 2 times slower than treepuzzle, although most $(90 \%+)$ of the CPU time was spent on inferring the witness quartet set through time-consuming process calls to fastDNAml. Currently we are adopting fastDNAml (as well as other ML techniques) directly into the $\mathrm{HC}^{*}$ source code, which should significantly speed up the run time of $\mathrm{HC}^{*}$. This enables $\mathrm{HC}^{*}$ to be practical on larger datasets (input set $n>100$ ). Moreover the witness quartet set lends itself naturally to be parallel computed should the need arise.

\section{Conclusion and Future Work}

This paper describes the $\mathrm{HC}^{*}$ method as an efficient method for recovery phylogenetic tree edges best supported by the witness quartet set. The $\mathrm{HC}^{*}$ method makes interesting accuracy guarantees on the edges recovered with respect to the witness quartet set. This focuses a larger phylogenetic inference problem into numerous, smaller subproblems on input size four. As more sophisticated and powerful ML methods arise on learning models of evolution from sequences (given fixed topologies or on small topology search spaces), ${ }^{17,14}$ the accuracy on inferring quartet topologies will continue to improve. Thus $\mathrm{HC}^{*}$ becomes a potentially powerful technique for approximing ML based phylogenetic tree inference.

Due to the efficient and optimal nature of $\mathrm{HC}^{*}$, one area of further investigation would be using $\mathrm{HC}^{*}$ to evaluate the effectiveness of weighted quartet methods. This stems of the motivation that some early studies ${ }^{15}$ suggests that unweighted quartet methods display poor scalability properties. In particular, it would be interesting to combine sophisticated inference techniques such as in Ref. 14 for inferring quartet 
topologies along with various schemes for constructing weighted quartets (e.g. ensemble learning) to further probe the scalability properties of weighted quartet methods using $\mathrm{HC}^{*}$ as a baseline study.

\section{References}

1. M. Blanchette, Algorithms for phylogenetic footprinting. In Proceedings of the 5th Conference on Research in Computational Molecular Biology (2001), ACM Press, pp. $49-58$.

2. D. Bryant, V. Berry, P. Kearney, M. Li, T. Jiang and T. Wareham, A practical algorithm for recovering the best supported edges of an evolutionary tree. In Proceedings of the Eleventh Annual ACM-SIAM Symposium on Discrete Algorithms (SODA) (2000), ACM Press, pp. 287-296.

3. Y. Cao, J. Adachi and M. Hasegawa, Comments on the quartet puzzling method for finding maximum likelihood tree topologies. Mol. Bio. Evol. 15 (1998), 87-89.

4. P. Erdos, K. Rice, M. Steel, L. Szekely and T. Warnow, The short quartet method. International Congress on Automata, Languages and Programming (1997).

5. N. Friedman, M. Ninio and I. Pe'er, A structural EM algorithm for phylogenetic inference. In Proceedings of the 5th Conference on Computational Molecular Biology (2001), ACM Press, pp. 132-140.

6. T. Jiang, P. Kearney and M. Li, A polynomial time approximation scheme for inferring evolutionary trees from quartet topologies and its application. SIAM Journal on Computing 30 (2001), 1942-1961.

7. T. Jiang and M. Zhang, Eds. Current Topics in Computational Molecular Biology. MIT Press, 2002.

8. B. Korber, M. Muldon, J. Theiler, F. Gao, R. Gupta, A. Lapedes, B. Hahn, S. Wolinsky and T. Bhattacharya, Timing the ancestor of the $\{\mathrm{HIV}-1\}$ pandemic strains. Science 288 (2000), 1789-96.

9. M. Kuhner and J. Felsenstein, A simulation comparison of phylogeny algorithms under equal and unequal evolutionary rates. Molecular Biology and Evolution 11 (1994), $459-468$.

10. G. Olsen, H. Matsuda, R. Hagstrom and R. Overbeek, Fastdnaml: a tool for construction of phylogenetic trees of dna sequences using maximum likelihood. Current Applications in Biosciences 10 (1994), 41-48.

11. A. Rambaut and N. and Grassly, Seq-gen: An application for the monte carlo simulation of dna sequence evolution along phylogenetic trees. Comp. Appl. Biosci. 13 (1997), 235-238.

12. J. Rogers and D. Swofford, A fast method for approximating maximum likelihoods of phylogenetic trees from nucleotide sequences. Systematic Biology 47 (1998), 77-89.

13. M. Rosenberg and S. Kumar, Incomplete taxon sampling is not a problem for phylogenetic inference. Proc Natl Acad Sci, USA 98(19) (2001), 10751-6.

14. E. Schadt, J. Sinsheimer and K. Lange, Computational advances in maximum liklihood methods for molecular phylogeny. Genome Research (1998), 222-233.

15. K. St. John, T. Warnow, M. Moret and L. Vawter, Performance study of phylogenetic methods: (unweighted) quartet methods and neighbour-joining. In Symposium on Discrete Algorithms (2001), ACM Press, pp. 196-205.

16. K. Strimmer and A. Haeseler, Quartet puzzling: a quartet maximum likelihood method for reconstructing tree topologies. Mol. Biol. Evol. 13 (1996), 964-969.

17. Z. Yang, Maximum likelihood analysis of adaptive evolution in hiv-1 gp120 env gene. In Pacific Symposium on BioComputing (2001), pp. 226-237. 